\title{
Risk Assessment Method of Coastal Erosion Disasters
}

Shiyong Wen, Fengshou Zhang, Xiang Wang, Xinxin Wang, Fei Li, Fei Wang, Ning Gao, Xuegong Xu

${ }^{1}$ National Marine Environment Monitoring Center, Dalian 116023, China

${ }^{2}$ College of Urban and Environmental Sciences \& Laboratory for Earth Surface Process of Ministry of Education, Peking University, Beijing 100871, China

\section{海岸侵蚀灾害风险评估技术方法}

文世勇, 张丰收, 王祥, 王新新, 李飞, 王飞, 高宁, 许学工 国家海洋环境监测中心海域资源环境动态监管中心, 辽宁 大连 116023 , 中国

\begin{abstract}
Coastal erosion disaster is one of marine disasters. Coastal erosion disasters caused large areas of land loss, destroyed coastal ecological environment system, and seriously affect the sustainable development of social economy in coastal areas. To effectively cope with the coastal erosion disasters prevention and emergency management needs, firstly, according to related research results, the connotation and the quantitative expression of risk assessment of coastal erosion disaster were expounded from the disaster risk assessment and disaster prevention and mitigation perspective. Secondly, the assessment model of hazard degree, vulnerability degree and disasters risk were set up respectively based on the coastal erosion disasters formation mechanism and its damage characteristic, which would provide the support for the risk assessment of coastal erosion disaster.
\end{abstract}

Keywords: Coastal erosion disasters; indexes; risk assessment; hazard assessment; vulnerability assessment

\section{摘要}

海岸侵蚀灾害是海洋地质灾害的一个灾种。海岸侵蚀 导致沿岸土地大面积流失, 破坏沿岸生态环境系统, 严重影响沿海地区社会经济的可持续发展。为有效应 对海岸侵蚀灾害防灾减灾与应急管理的需求, 在参考 相关研究成果的基础上, 从灾害风险评估与防灾减灾 的视角, 阐述了海岸侵蚀灾害风险评估的内涵及其定
量表达式; 根据海岸侵蚀灾害成因机制和危害特点, 分别建立了海岸侵蚀灾害危险度、承灾体易损度及风 险评估模型, 为实现海岸侵蚀灾害风险评估提供支 持。

关键词: 海岸侵蚀灾害; 指标体系; 风险评估; 危险 度；易损度

\section{1. 引言}

海岸侵蚀（Coastal erosion）是指灾害海洋动力作用 下, 导致海岸线向陆地迁移或潮间带滩涂和潮下带底 床下蚀的海岸变化过程[1]。海岸侵蚀灾害造成房屋 和道路冲毁, 海滩减少和毁坏, 沿岸工程冲毁, 土地 大面积流失, 旅游设施受损, 给沿岸人民的生产、生 活及生态环境系统带来严重影响或构成潜在的威胁 [2]。如何科学有效地开展海岸侵蚀灾害防灾减灾与 灾害管理, 是当前急需解决的关键问题。

灾害风险评估是灾害防灾减灾管理和灾害应急 响应的重要内容之一。当前国际灾害防灾减灾理念由 “灾后救援” 向 “灾前预防” 的转变, 将灾害在发生 前消灭于萌芽中, 使灾害损失尽量最小化, 有效地减 轻灾害对社会经济造成的损失[3]。自然灾害风险一 直是国际社会关注的重点领域之一, 联合国认为 “风 险评估是采取充分和成功减灾政策和措施的必要步 骤”, 是作为灾害防灾减灾的一个首要原则。随着国 内外减灾领域对防灾减灾各方面问题认识的不断深 化和国际减轻自然灾害十年 (IDNDR) 活动的逐步 深入与扩展, 自然灾害风险分析与风险管理工作在防 灾减灾中的作用和地位日益突现[3]。

目前, 海岸侵蚀灾害风险研究工作主要集中在海 岸侵蚀现状、海岸侵蚀灾害发生机制和海岸侵蚀未来 预测分析这三个方面研究工作。海岸侵蚀现状主要是 
基于现场调查资料来分析当前调查岸段的侵蚀状况 [2,4 16]; 海岸侵蚀灾害发生机制主要是通过外业调 查手段, 获取海岸侵蚀强度, 分析其发生机制 [6,17 28]; 以及未来预测分析 [29 41]。从灾害风险 评估视角开展海岸侵蚀风险评估研究工作较少, 如张 晓龙[42]定性分析了海面上升、风暴潮、黄河断流等 灾害风险因素作用下黄河三角洲海岸侵蚀状况; Del Río 等[43]建立了包含 11 个危险性评估指标和 6 个承 灾体易损度评估指标的指标体系, 通过对各指标分 级, 并采用加权平均法进行综合评价, 给出海岸侵蚀 灾害风险值。然而, 这些海岸侵蚀灾害评估多侧重于 某一方面, 或侧重致灾因子的危险性评价, 或侧重承 灾体的易损性评价, 缺乏从灾害风险评估的角度来研 究海岸侵蚀灾害。

本文研究的目的是在已有的相关研究 [44 46]的 基础上, 从灾害风险评估、灾害防灾减灾视角, 明确 海岸侵蚀灾害风险评估的内涵及其定量表达式, 分别 建立了海岸侵蚀灾害危险度、承灾体易损度及风险评 估模型, 为海岸带防灾减灾提供科学支撑, 有效保障 海岸带资源环境健康与沿海地区社会经济的可持续 发展。

\section{2. 海岸侵蚀灾害风险评估理论及定量表达}

不同的学者对灾害风险的认识和表达有一定的差异。 IPCC 的报告指出风险是灾害发生概率与其对承灾体 影响程度的乘积 [47]。Crichton 提出风险取决于 3 个 因素: 致灾因子、脆弱性和暴露性[48]。联合国 ISDR 指出风险是致灾因子、脆弱性和恢复力的函数 [49-50]。但无论以何种方式表达, 灾害风险评估最核 心的内涵为风险是灾害危险度与承灾体易损度的函 数, 即:

$$
R=f(H, V)
$$

其中, $R$ 为灾害风险; $H$ 为灾害危险度, 即灾害 发生的可能性, 取值从 0 到 $1 ; V$ 为承灾体易损度, 即承灾体遭受灾害危害的可能损失。

依据联合国赈灾组织和联合国人道主义事务部 公布的自然灾害风险评估理论和借鉴其他自然灾害 风险评估的先进思想, 结合海岸侵蚀对不同类型承灾 体造成的危害特点, 将海岸侵蚀灾害风险评估定义为 “在一定区域和给定时段内, 对评估区内海岸侵蚀灾 害有可能发生的后退范围 (后退速率) 及其可能造成 的损失的评估”, 包括海岸侵蚀灾害危险度和承灾体 易损度评估两个内容。海岸侵蚀灾害危险度, 即海岸 侵蚀灾害有可能发生的后退范围 (后退速率), 是指
在一定区域和给定时段内, 通过对孕灾环境因子与致 灾因子的关系分析, 定量获取评估区域内海岸有可能 发生的后退范围 (后退速率), 反映灾害的自然属性; 承灾体易损度, 即承灾体有可能发生的损失, 是指在 一定区域和给定时段内, 由于海岸侵蚀有可能导致的 该区域内所存在的一切人和财物的潜在最大破坏程 度或潜在最大损失程度。

$$
R=\alpha \times H \times V
$$

其中, $R$ 为海岸侵蚀灾害风险; $\alpha$ 为岸线自身的 脆弱性, 由 Delphi 法来确定; $H$ 为海岸侵蚀灾害危 险性; $V$ 为海岸侵蚀灾害承灾体易损度。

如何定量表达海岸侵蚀灾害危险性 $H$ 和承灾体 易损性 $V$ 是实现海岸侵蚀灾害风险评估的关键。

\section{3. 海岸侵蚀灾害危险度评估方法}

3.1. 基于致灾因子的海岸侵蚀灾害危险度评估方法 造成海岸侵蚀灾害的原因多种多样, 但基本可归纳为 自然因素和人为因素两大类。其中, 自然因素包括: 风暴潮、海浪侵袭, 海平面上升, 河流输沙减少; 人 为因素包括: 海岸采砂, 河流水利工程拦截泥沙, 海 岸工程加强了水动力, 海滩植被的破坏等[44]。而在 这些影响因素中, 风暴潮、海浪, 海平面上升, 河流 入海泥沙量减少, 人类活动四个方面是造成海岸侵蚀 最主要的因素[44]。因此, 基于致灾因子的海岸侵蚀 灾害危险度评估方法为:

$$
H=\sum_{i=1}^{n} H_{i}
$$

其中, $H$ 为海岸侵蚀灾害危险度; $H_{i}$ 为表示第 $\mathrm{i}$ 个指标产生的危险度, 如针对砂质岸线, 根据 Bruun 定律 [51], 由海平面上升的海岸侵蚀灾害危险度评估 模型为:

$$
H_{s}=\frac{L}{B+d} \times S \times G
$$

其中, $H s$ 为岸线后退量, $S$ 为海面相对上升量; $G[52]$ 为侵蚀泥沙仍然在活动剖面上的比例, 砂质海 岸 $G$ 为 1 , 而对碎石滩 $G$ 假定为 $0.5 ; d$ 为闭合水深, $L$ 为从水深 $d$ 到岸线的距离, $B$ 为滩肩高度 (或其他 侵蚀区高程估计)。

根据 Krieble 等[53]研究结果, 由风暴潮引起的 海岸侵蚀危险度评估模型（经验公式）为:

$$
H_{w}=h_{m i} S_{m i}\left(\frac{t_{d i}}{12}\right)^{0.3}
$$


Risk Analysis and Crisis Response in Big Data Era (RAC-16)

其中, $H_{w}$ 为修正后的某次风暴潮引起的海岸后 退量 $(\mathrm{m}) ; h_{m i}$ 为某次风暴潮的最大波高 $(\mathrm{m}) ; S_{m i}$ 为某次风暴潮的最大增水 $(\mathrm{m}) ; t_{d i}$ 为某次风暴潮持 续的时间 (h)。

若只考虑海平面上升和风暴潮这两个要素, 该海 岸侵蚀灾害危险度为上述二者之和。

3.2. 基于遥感技术的海岸侵蚀灾害危险度评估方法

常规海岸调查在资料获取、信息处理、多时相同步观 测等方面存在较大局限性。遥感技术是运用物理手

段、数学方法和地学规律的一项现代综合性探测技 术, 具有探测范围广、采集数据快, 能动态反映地面 事物的变化及获取的数据具有综合性等优势特点, 因 此遥感技术在获取海岸不同尺度和不同层次的连续、 动态信息是一种非常有效的手段。

基于遥感技术的海岸侵蚀灾害危险度评估方法 主要是利用多期高分辨率的不同时期的遥感影像数 据, 在完成图像校正等预处理工作的基础上, 通过提 取不同时期的岸线，在 GIS 平台下利用数字岸线分 析系统模块 (Digital Shoreline Analysis System, DSAS）分析模块建立监测岸段后退速率趋势模型, 同时在无人为因素干扰情况下预测评估未来时段内 有可能产生的侵蚀范围[46]:

$$
\begin{aligned}
& x=x_{\mathrm{int}}+\frac{L R R \times \Delta t}{D I S} \times\left(x_{\mathrm{int}}-x_{\text {sta }}\right) \\
& y=y_{\mathrm{int}}+\frac{L R R \times \Delta t}{D I S} \times\left(y_{\mathrm{int}}-y_{\text {sta }}\right)
\end{aligned}
$$

其中, $(x, y)$ 为预测点的坐标, 单位为 $\mathrm{m} ;\left(x_{i n t}, y_{i n t}\right)$ 为节点的坐标, 单位为 $\mathrm{m}$; $\left(x_{\text {sta }}, y_{\text {sta }}\right)$ 为基线的坐标, 单位为 $\mathrm{m} ; L R R$ 为岸线变化速率, 单位为 $\mathrm{m} / \mathrm{yr} ; \Delta t$ 为变化时间, 单位为 $\mathrm{yr} ; D I S$ 为节点与基线的距离, 单位为 $\mathrm{m},\left(x_{i n t}, y_{i n t}\right) 、\left(x_{\text {sta }}, y_{\text {sta }}\right) 、 \mathrm{LRR} 、 \mathrm{DIS}$ 的值可以 从 DSAS 分析模块得到的结果获取。

\section{4. 承灾体易损性评估方法}

承灾体易损度评估指标是指由于海岸侵蚀的发生, 导 致承灾体的破坏而产生的经济损失 (后果)。在参考 大量相关文献材料的基础上, 根据完整性和可量化的 承灾体分类原则, 结合海岸侵蚀灾害的危害特点, 确 定了海岸侵蚀灾害承灾体因子及分类体系编码 [44], 并根据承灾体易损度的内涵, 建立承灾体易损度评估 指标[44]。

承灾体易损度包含承灾体价值和承灾体易损的 程度两方面内容, 即:

$$
V=\omega \times E
$$

其中, $V$ 为易损度, $\omega$ 为不同危险度条件下, 承 灾体的易损程度, 即海岸侵蚀灾害承灾体易损性, 由 易损性曲线确定, $E$ 为各类承灾体单价经济价值。

\section{5. 结 语}

灾害风险评估是灾害防灾减灾与应急管理的基础。本 文在参考相关文献的基础上, 根据当前国际通行的灾 害减灾防灾理念, 借鉴其他自然灾害风险评估先进思 想, 参考海岸侵蚀相关资料, 结合海岸侵蚀灾害成灾 机制和危害特点, 初步定义了海岸侵蚀灾害风险评 估、海岸侵蚀灾害危险度、承灾体易损度的内涵, 为 开展海岸侵蚀灾害风险评估提供理论指导; 根据海岸 侵蚀灾害成灾机制和危险度评估指标, 建立了包括海 平面上升、风暴潮等多种要素的海岸侵蚀灾害危险度 评估模型; 根据海岸侵蚀灾害危害特点和承灾体易损 度评估指标, 建立了以经济价值为依据的承灾体易损 度评估模型; 并依据海岸侵蚀灾害风险评估内涵, 建 立了海岸侵蚀灾害风险评估模型, 为实现海岸侵蚀灾 害风险评估提供模型方法。

本文从灾害防灾减灾与应急管理的视角, 初步提 出海岸侵蚀灾害风险评估理论、方法体系, 期望海岸 侵蚀灾害风险评估理论、技术方法不断完善与深化, 为评估区海岸侵蚀灾害防灾减灾, 合理规划沿岸产业 布局提供技术支持。

\section{Acknowledgements}

This study was financial supported by the operational work of State Oceanic Administration of coastal erosion disasters survey and assessment, and the National Natural Science Foundation of China (NO. 41271102, 40830746).

\section{致谢}

本研究得到国家海洋局海岸侵蚀灾害调查和损失评 估工作业务、国家自然科学基金项目（NO.41271102， 40830746）的资助。

\section{参考文献}

[1] 陈吉余, 夏东兴, 虞志英, 蔡锋. 中国海岸侵蚀 概要. 北京: 海洋出版社, 2010.

[2] 王玉广, 李淑媛, 苗丽娟. 辽东湾两侧砂质海岸 侵蚀灾害与防治. 海岸工程, 2005, 24(1):9 18.

[3] 张俊香.新一代自然灾害风险区划原理与方法研 
究——以地震灾害为例. 北京: 北京师范大学资 源学院, 2005 .

[4] 夏东兴, 王文海.中国海岸侵蚀述要. 地理学报, 1993，48(5):468 476.

[5] Davidson-Arnott R G D, Ollerhead J. Nearshore erosion on a cohesive shoreline. Marine Geology, 1995, 122(4):349-365.

[6] 苗丰民, 庄振业. 辽东湾东部砂岸的近期变化及 演变趋势. 海洋学报(中文版), 1996,18(2):74-84.

[7] List J H, Jr Sallenger A H, Hansen M E, Jaffe B E Accelerated relative sea-level rise and rapid coastal erosion: testing a causal relationship for the Louisiana barrier islands. Marine Geology, 1997, 140(3-4):347-365.

[8] Andriani G F, Walsh N. Rocky coast geomorphology and erosional processes: A case study along the Murgia coastline South of Bari, Apulia-SE Italy. Geomorphology, 2007, 87(3):224-238.

[9] 王玉广, 张宪文, 贾凯, 王权明. 辽东湾绥中海 岸侵蚀研究. 海岸工程, 2007, 26(1):1 5.

[10] 孔繁德. 秦皇岛市海岸侵蚀研究及防治对策. 城 市发展研究，2008，15(1):73 76。

[11] Jabaloy-Sánchez A, Lobo F J, Azor A, Bárcenas P, Fernández-Salas L M, del Río V D, Pérez-Peña L $\mathrm{V}$. Human-driven coastline changes in the Adra River deltaic system, southeast Spain. Geomorphology, 2010, 119 (1-2): 9-22.

[12] 徐宗军, 张绪良, 张朝晖. 山东半岛和黄河三角 洲的海岸侵蚀与防治对策. 科技导报, 2010, 28(10):90 95.

[13] CUI B L, LI X Y. Coastline change of the Yellow River estuary and its response to the sediment and runoff (1976-2005). Geomorphology, 2011, 127 (1-2):32-40.

[14] Rahman A F, Dragoni D, El-Masri B. Response of the Sundarbans coastline to sea level rise and decreased sediment flow: A remote sensing assessment. Remote Sensing of Environment, 2011, 115(12):3121-3128.

[15] Liu Y, Huang H, Qiu Z, Fan J. Detecting coastline change from satellite images based on beach slope estimation in a tidal flat. International Journal of Applied Earth Observation and Geoinformation, 2013, 23:165-176.

[16] 庄振业, 杨燕雄, 刘会欣. 环渤海砂质岸侵蚀和 海滩养护. 海洋地质前沿，2013，29(2):1-9

[17] 王文海. 我国海岸侵蚀原因及其对策. 海洋开 发, 1987, 1:8 12.

[18] 王文海, 吴桑云. 山东省海岸侵蚀灾害研究. 自 然灾害学报，1993，2(4):60 66.

[19] 王颖, 吴小根. 海平面上升与海滩侵蚀. 地理学 报，1995，50(2):118 127.

[20] 季子修. 中国海岸侵蚀特点及侵蚀加剧原因分 析. 自然灾害学报, 1996, 5(2):69 79.

[21] Budetta P, Galietta G, Santo A. A methodology for the study of the relation between coastal cliff erosion and the mechanical strength of soils and rock masses. Engineering Geology, 2000,
56(3-4):243-256.

[22] Zhang K Q, Douglas B, Leatherman S. Do storms cause long-term beach erosion along the U.S. East Barrier Coast?. Journal of Geology, 2002, 110 (4):493 502

[23] 丰爱平, 夏东兴, 谷东起, 吴桑云, 李朝新. 莱 州湾南岸海岸侵蚀过程与原因研究. 海洋科学 进展, 2006, 24(1):83 90.

[24] 温倩, 方风满, 高健. 山东半岛海岸侵蚀成因及 防治对策研究. 国土与自然资源研究, 2006, 1:52 53.

[25] 张晓龙, 李培英. 现代黄河三角洲的海岸侵蚀及 其环境影响。海洋环境科学, 2008, 27(5):475 479.

[26] Brunel C, Sabatier F. Potential influence of sea-level rise in controlling shoreline position on the French Mediterranean Coast. Geomorphology, 2009, 107:47-57.

[27] HUANG G. Time lag between reduction of sediment supply and coastal erosion. International Journal of Sediment Research, 2011, 26 (1):27-35.

[28] 郭婷婷, 邵晓昕. 浅析海岸带侵蚀. 环境保护与 循环经济，2011，(5):37 39+59.

[29] 杨燕雄, 贺鹏起, 谢亚琮, 吴青, 马爱民. 秦皇 岛海岸侵蚀研究. 中国地质灾害与防治学报, 1994, 5(S1):166 170.

[30] 陈坚, 谢在团. 未来海平面上升对厦门海滩侵蚀 的测算. 台湾海峡, 1998, 17 (4):391-395.

[31] Leont'Yev I O. Modeling erosion of sedimentary coasts in the western Russian Arctic. Coastal Engineering, 2003, 47(4):413-429.

[32] Walkden M J A, Hall J W. A predictive Mesoscale model of the erosion and profile development of soft rock shores. Coastal Engineering, 2005, 52 (6):535-563.

[33] Brown I, Jude S, Koukoulas S, Nicholls R, Dickson M, Walkden M. Dynamic simulation and visualisation of coastal erosion. Computers, Environment and Urban Systems, 2006, 30(6):840-860.

[34] Callaghan D P, Nielsen P, Short A, Ranasinghe R. Statistical simulation of wave climate and extreme beach erosion. Coastal Engineering, 2008, 55(5):375-390.

[35] Callaghan D P, Roshanka R, Andrew S. Quantifying the storm erosion hazard for coastal planning. Coastal Engineering, 2009, 56(1): 90-93.

[36] 韩晓庆, 高伟明, 禇玉娟. 河北省自然状态沙质 海岸的侵蚀及预测. 海洋地质与第四纪地质, 2008, 28(3):23 29.

[37] Hapke C, Plant N. Predicting coastal cliff erosion using a Bayesian probabilistic model. Marine Geology, 2010, 278 (1-4):140-149.

[38] Trenhaile A S. Modeling cohesive clay coast evolution and response to climate change. Marine Geology, 2010, 277(1-4):11-20.

[39] Kane H H, Fletcher C H, Romine B M, Anderson T R, Frazer N L, Barbee M M. Vulnerability assessment of Hawai'i's cultural assets attributable 
Risk Analysis and Crisis Response in Big Data Era (RAC-16)

to erosion using shoreline trend analysis techniques. Journal of Coastal Research, 2012, 28(3):533-539.

[40] Loureiro C, Ferreira Ó, Cooper J A G. Extreme erosion on high-energy embayed beaches: Influence of megarips and storm grouping. Geomorphology, 2012, 139-140:155-171.

[41] Ranasinghe R, Callaghan D, Stive M J F. Estimating coastal recession due to sea level rise: beyond the Bruun rule. Climatic Change, 2012, $110(3-4): 561-574$

[42] 张晓龙, 李培英. 黄河三角洲滨海湿地海岸侵蚀 灾害及风险. 应用基础与工程科学学报, 2006, 14 (S1):247 252.

[43] Del Río L, Gracia F J. Erosion risk assessment of active coastal cliffs in temperate environments. Geomorphology, 2009, 112(1-2): 82-95.

[44] XU Lifen, WEN Shiyong, ZHAO Dongzhi, XU Xuegong. On the coastal erosion risk assessment indexes. Journal of Risk Analysis and Crisis Response, 2013, 3(3):146-155.

[45] 包春霞, 文世勇, 徐丽芬, 吴䑣, 赵冬至, 黄凤 荣, 许学工. 基于海平面上升的辽东湾绥中海岸 侵蚀危险度评估. 灾害学, 2015,30(1): 205-210。

[46] Wen Shiyong, Zhang Fengshou, Wang Xiang, Wang Zizhu, Li Fei, Wang Fei, Wang Xinxin. Hazard degree assessment of coastal erosion at Tianlongsi adjacent sandy coast. The Fourth International Workshop on Earth Observation and Remote Sensing Applications (EORSA 2016), IEEE, Guangzhou, China, July 4-6, 2016.

[47] IPCC (Intergovernmental Panel on Climate Change).Technical summary: climate change 2001: impacts, adaptation, and vulnerability. A Report of Working Group II of the Intergovernmental Panel on Climate Change. Cambridge: Cambridge University Press, 2001.

[48] Crichton D. The risk triangle // Ingleton J (Ed.): Natural Disaster Management. London: Tudor Rose, 1999: 102-103.

[49] Bradshaw S. Handbook for Estimating the Socio-economic and Environmental Effects of Disasters. United Nations, Economic Commission for Latin America and the Caribbean \& International Bank for Reconstruction and Development, 2003.

[50] UNISDR (United Nations Office for Disaster Risk Reduction).Living with Risk A global review of disaster reduction initiatives. New York and Geneva, UNISDR, 2004.

[51] Bruun P F. Sea-level rise as a cause of shore erosion. Journal of the Waterways and Harbors Division, 1962, 88: 117-132.

[52] Snoussi M, Ouchani T, Khouakhi A, Niang-Diop I Impacts of sea-level rise on the moroccan coastal zone: quantifying coastal erosion and flooding in the tangier bay. Geomorphology, 2009, 107 (1-2): $32-40$.

[53] Kriebel D, Dalrymple R, Pratt A, Sakovich V. A shoreline risk index for northeasters. Proceedings of ASCE Int. Conf. on Natural Disaster Reduction, 